\title{
Sedentary behaviour, physical activity, cardiorespiratory fitness and cardiometabolic risk in psychosis: The PsychiActive project
}

\author{
Javier Bueno-Antequera, Miguel Ángel Oviedo-Caro, Diego Munguía-Izquierdo *
}

Department of Sports and Computer Science, Section of Physical Education and Sports, Faculty of Sports Sciences, Universidad Pablo de Olavide, ES-41013 Seville, Spain

\section{A R T I C L E I N F O}

\section{Article history:}

Received 2 July 2017

Received in revised form 29 September 2017

Accepted 8 October 2017

Available online $\mathrm{xxxx}$

\section{Keywords:}

Psychosis

Sedentary lifestyle

Physical activity

Cardiorespiratory fitness

Metabolic disease

\begin{abstract}
A B S T R A C T
This study aimed to explore the possible independent associations of sedentary behaviour (SB), physical activity (PA), and cardiorespiratory fitness (CRF) with clustered (CCRS) and individual cardiometabolic risk (waist circumference [waist], systolic/diastolic blood pressure, triglycerides, high-density lipoprotein-cholesterol, and fasting blood glucose) in patients with psychosis. In 43 outpatients with psychosis (mean age \pm SD: $42.3 \pm$ 8.5 years, 86\% men), SB and light, moderate-to-vigorous, and total PA were measured with the SenseWear Pro3 Armband, and CRF with the 6-minute walking test. Multiple linear regression models adjusted for multiple confounders were applied. High SB, low PA and low CRF levels were associated with an unfavourable cardiometabolic risk profile (increased presence of metabolic syndrome and number of cardiometabolic abnormalities, as well as worse values and elevated presence of abnormalities for all individual cardiometabolic risk factors). SB was associated with CCRS, number of cardiometabolic abnormalities, waist, and fasting blood glucose (all $\mathrm{p}<0.05$ ). After adjusting for PA and CRF, waist and fasting blood glucose remained significant. Light PA was associated with waist, moderate-to-vigorous PA with CCRS, and total PA with CCRS and waist (all p $<0.05$ ). These results became non-significant after adjusting for SB and CRF. CRF was associated with CCRS, waist, and systolic blood pressure (all $\mathrm{p}<0.05$ ). The associations with CCRS and waist remained significant after adjusting for SB and PA. Together, these results suggest the importance of considering SB and CRF, regardless PA, in the prevention and treatment of cardiometabolic disorders among patients with psychosis.
\end{abstract}

(c) 2017 Elsevier B.V. All rights reserved.

\section{Introduction}

Patients with psychosis, including schizophrenia and bipolar disorders (World Health Organization, 1992), have a greatly reduced life expectancy, up to 15 years, compared to the general population (Lawrence et al., 2013), with cardiometabolic disease being the main contributor (Correll et al., 2017). The increased prevalence of metabolic syndrome and cardiometabolic abnormalities is also evident (Vancampfort et al., 2015b), and has become a major health challenge. Of concern, a recent study (Bruins et al., 2017) revealed that cardiometabolic risk factors remain seriously undertreated in people with psychosis and, therefore, better prevention and treatment of metabolic disorders are imperative for reducing the overwhelming risk of premature mortality.

In general population, there is an established-evidence base indicating that, independently, less sedentary behaviour (SB) and greater physical activity (PA) decrease cardiometabolic risk (Biswas et al.,

\footnotetext{
* Corresponding author at: Departamento de Deporte e Informática, Universidad Pablo de Olavide, Carretera Utrera Km. 1, s/n, 41013 Sevilla, Spain.

E-mail addresses: jbueant@upo.es (J. Bueno-Antequera), maovicar@upo.es (M.Á. Oviedo-Caro), dmunizq@upo.es (D. Munguía-Izquierdo).
}

2015). Two meta-analyses (Stubbs et al., 2016a; Stubbs et al., 2016b) highlighted that patients with psychosis engage in more SB and in less PA than the general population. To date, some studies (e.g., (Nyboe et al., 2015; Stubbs et al., 2015; Vancampfort et al., 2015a)) have suggested associations of SB and PA with cardiometabolic risk in patients with psychosis. While helpful, almost all of these studies have relied upon self-report measures, which introduce bias (Soundy et al., 2014), and only one study examined the independent associations of SB and PA with cardiometabolic risk (Stubbs et al., 2017). In this regard, more research, as well as the preferential use of objective measures, is necessary to improve our understanding of the independent effects of these two exposures on cardiometabolic health in this population.

There is also a firmly established-base indicating that a low cardiorespiratory fitness (CRF) level is a strong independent predictor of allcause and cardiovascular mortality (Harber et al., 2017), with two recent studies (Knaeps et al., 2016a; Knaeps et al., 2016b) finding that CRF mediates the association of SB and PA with clusteredcardiometabolic risk and its individual-components. Patients with psychosis have significantly lower CRF compared with controls (Vancampfort et al., 2017), and the independent associations of SB, PA, and CRF with clustered-cardiometabolic risk and individualcardiometabolic risk factors remain unexplored. 
The aim of this study was to explore the possible independent associations of SB, PA, and CRF with clustered-cardiometabolic risk and individual-cardiometabolic risk factors in patients with psychosis (schizophrenia and bipolar disorders).

\section{Methods}

\subsection{Participants and setting}

Adults with a diagnosis of psychotic illness including schizophrenia and bipolar disorders according to ICD-10 criteria and stabilized on antipsychotic medication was recruited from 11 different outpatient mental healthcare settings in southern Spain. Patients were excluded if they had clinical instability, co-morbid substance abuse, or evidence of uncontrolled cardiovascular, neuromuscular and endocrine disorders. Participants received a full-fasting laboratory screening and anthropometric measurement, performed a walk test, wore a multisensor armband, and completed questionnaires about sociodemographic characteristics and symptomatology. Patient's medical records were also registered. The study procedure was approved by the Universidad Pablo de Olavide Ethics Committee. All patients gave their informed written consent prior to enrolling in the study and after receiving information about the aims and protocol. There was no compensation for participation.

\section{2. $S B$ and $P A$}

SB and PA were obtained with a SenseWear Pro3 Armband (BodyMedia Inc., Pittsburgh, PA, USA), a device to accurately estimate energy expenditure (Johannsen et al., 2010). Patients were required to wear the SenseWear on their left arm triceps muscle for nine consecutive days, $24 \mathrm{~h} /$ day, except when showering or swimming. The first and last days were excluded from the analysis to minimize the Hawthorne effect (i.e., "a general scientific fact that the process of observation alters the phenomenon being observed") (Corder et al., 2008). Seven days of recordings with a minimum of 1368 min of registration per day was necessary to be included in the analysis. Energy expenditure was estimated using data recorded from multiple sensors and using specific-algorithms developed by the manufacturer (SenseWear Professional software, version 8.1). Time spent in SB $(1.0<$ MET $\leq 1.5)$ and PA intensities (light, $1.5<\mathrm{MET} \leq 3.0$; moderate-to-vigorous, $>3.0$ MET; and total $>1.5 \mathrm{MET}$ ) was derived using the measured MET values during waking hours.

\section{3. $C R F$}

CRF was assessed using the 6-minute walking test according to Rikli and Jones (1999) in an indoor course with a flat, firm surface and with minimal external stimuli. Patients were instructed to walk as far as possible during a 6-minute period around a 45.7-meter rectangular course delimited by cones, without running or jogging. Resting was allowed if necessary, but walking was to be resumed as soon as possible. Standardized-encouragements were used at recommended intervals (Rikli and Jones, 1999). The same trained instructor explained the protocol, gave a demonstration prior to the start, supervised the test and recorded the total distance walked to the nearest $0.1 \mathrm{~m}$ for each patient. The 6-minute walking test has been shown to be a reliable and valid method to assess CRF in patients with psychosis (Gomes et al., 2016).

\subsection{Cardiometabolic risk}

The cardiometabolic risk factors were collected by trained-staff in the morning after an overnight fast including waist circumference (waist), systolic/diastolic blood pressure, triglycerides, high-density lipoprotein-cholesterol, and fasting blood glucose. Waist was measured to the nearest $0.1 \mathrm{~cm}$ using a measuring tape (Harpenden
Anthropometric Tape; Holtain, Dyfed, UK) placed at the midpoint between the last rib and the iliac crest. Blood pressure was measured in a seated position after 10-minute rest period with an electronic monitor (Omron Healthcare Europe BV, Hoofddorp, The Netherlands) placed on the left arm wrist. The mean of the two measures was used for analysis. If the two measures differed by $>1 \%$ for waist, $>20 \mathrm{~mm} \mathrm{Hg}$ for systolic and $>10 \mathrm{~mm} \mathrm{Hg}$ for diastolic blood pressure, a third measure was taken, and the median of the three was used for analysis (Ward and Anderson, 1998). The presence of metabolic syndrome and cardiometabolic abnormalities was assessed using the International Diabetes Federation criteria (Alberti et al., 2006). Additionally, a clusteredcardiometabolic risk score (CCRS) was constructed. The standardizednormalized indexes $(\mathrm{z}$-score $=[$ value - mean $] /$ standard deviation $)$ for blood pressure ([systolic + diastolic blood pressure] / 2), triglycerides, fasting blood glucose, waist, and the inverse of high-density lipoprotein cholesterol were summarized and divided by the number of variables included $(x=5)$ to generate the CCRS. Scores above zero represent higher cardiometabolic risk.

\subsection{Severity of psychiatric symptoms}

Severity of psychiatric symptoms during the previous week was assessed using the Brief Symptoms Inventory-18 (Derogatis, 2001), which has been recommended in patients with mental illness (Prinz et al., 2013). Scores range 0-72, with higher scores indicating a higher severity.

\subsection{Demographic, illness-related, and medication data}

Marital, educational, occupational and smoking status were selfreported. Weight and height were measured with to the nearest $0.1 \mathrm{~kg}$ and $0.1 \mathrm{~cm}$ using a scale (TANITA BC-420; Tanita, Tokyo, Japan) and stadiometer, respectively, and body mass index was calculated. Age, diagnosis, illness duration, and medication were retrieved from the patients' medical records, and antipsychotic medication was converted into daily equivalent dosages of chlorpromazine (Gardner et al. 2010).

\subsection{Statistical analysis}

Due to the skewed distributions, the analyses included the logarithmically transformed data of moderate-to-vigorous PA, triglycerides, and illness duration, as well as the reciprocally transformed data of fasting blood glucose and the square root-transformed data of chlorpromazine and severity of psychiatric symptoms. Differences in SB, PA, and CRF between metabolic syndrome presence were tested using Student's $t$-test. Patients were divided into groups according to high or low levels of SB, PA (light, moderate-to-vigorous and total), and CRF using the median splits, while Student's t, Chi-square, and Fisher exact tests were applied to establish differences. Pearson correlation coefficients were calculated between SB, PA, CRF, and cardiometabolic risk. Multiple linear regression analyses were performed with the cardiometabolic risk outcomes as dependent variables and SB, PA, and CRF as the independent variables. Model-1 was adjusted for gender, age, smoking, education, severity of psychiatric symptoms, illness duration, and chlorpromazine dose. Waist was added in Model-2. Additionally, SB, PA, and CRF, as applicable, were added in the fully adjusted models. Only patients with a complete dataset were included in the regression analysis. Residuals were tested for homoscedasticity, linearity and independence. Other than when light and total PA were simultaneously used as independent variables, the variance inflation factor never exceeded five, indicating that multi-collinearity was not a concern (Montgomery et al., 2012). The data were analysed using SPSS Statistics for Windows, Version 22.0 (Armonk, NY: IBM Corp), with statistical significance set at $\mathrm{p}$-value $<0.05$. Statistical comparisons between the two psychiatric 
groups were not carried out because the small sample size for bipolar disorders $(n=11)$ data could have led to type II statistical errors.

\section{Results}

Forty-three patients with psychosis were included in the analysis, and characteristics are summarized in Table 1. Within the sample, 28 patients $(65.2 \%)$ met the criteria for metabolic syndrome. Those with metabolic syndrome were significantly more sedentary, less physically active (for all PA variables), and had lower CRF than those without metabolic syndrome (all $\mathrm{p}<0.05$; data not shown). As presented in Table 2 , the high SB, low PA (all variables) and CRF groups exhibited a higher presence of metabolic syndrome and number of cardiometabolic abnormalities than their counterparts, yet not reaching statistical significance except when comparing light PA levels (both $\mathrm{p}<0.05$ ). Furthermore, worse values and a higher presence of abnormalities for all individualcardiometabolic risk factors were found in the high SB and low light

Table 1

Patients' characteristics $(n=43)$.

\begin{tabular}{|c|c|}
\hline Variables & Values \\
\hline Age (years) & $42.3 \pm 8.5$ \\
\hline Body mass index $\left(\mathrm{kg} / \mathrm{m}^{2}\right)$ & $30.5 \pm 5.5$ \\
\hline SB (h/day, \% of waking time) & $8.8 \pm 2.1(59)$ \\
\hline LPA (h/day, \% of waking time) & $4.3 \pm 1.6(28)$ \\
\hline MVPA (h/day, \% of waking time) & $1.9 \pm 1.2(13)$ \\
\hline TPA (h/day, \% of waking time) & $6.2 \pm 2.3(41)$ \\
\hline CRF (m) & $598.7 \pm 94.6$ \\
\hline MetS $^{a}$ & $28(65.1)$ \\
\hline No. of meeting MetS ${ }^{a}$ & $2.8 \pm 1.7$ \\
\hline Waist (cm) & $105.6 \pm 16.5$ \\
\hline IDF criteria & $36(83.7)$ \\
\hline $\mathrm{SBP}(\mathrm{mm} \mathrm{Hg})$ & $126.3 \pm 17.8$ \\
\hline IDF criteria & $21(48.8)$ \\
\hline $\mathrm{DBP}(\mathrm{mm} \mathrm{Hg})$ & $82.4 \pm 11.0$ \\
\hline IDF criteria & $20(46.5)$ \\
\hline $\mathrm{TG}(\mathrm{mg} / \mathrm{dL})$ & $210.5 \pm 177.0$ \\
\hline IDF criteria & $25(58.1)$ \\
\hline HDL-C (mg/dL) & $44.4 \pm 11.8$ \\
\hline IDF criteria & $21(48.8)$ \\
\hline FBG (mg/dL) & $106.9 \pm 38.8$ \\
\hline IDF criteria & $20(46.5)$ \\
\hline Severity of psychiatric symptoms $(0-72)^{\mathrm{b}, \mathrm{c}}$ & $14.7 \pm 11.6$ \\
\hline Illness duration (years) ${ }^{\mathrm{b}}$ & $16.2 \pm 9.3$ \\
\hline Chlorpromazine equivalent dose $(\mathrm{mg} / \text { day })^{\mathrm{b}}$ & $643.7 \pm 576.2$ \\
\hline Smoking status (current smoker) & $25(58.1)$ \\
\hline Gender (women) & $6(14.0)$ \\
\hline Race (Caucasian) & $43(100)$ \\
\hline \multicolumn{2}{|l|}{ Diagnoses } \\
\hline Schizophrenia spectrum disorders & $32(74.4)$ \\
\hline Bipolar disorders & $11(25.6)$ \\
\hline \multicolumn{2}{|l|}{ Marital status } \\
\hline Married & $6(14.0)$ \\
\hline Unmarried & $32(74.4)$ \\
\hline Separated/divorced & $5(11.6)$ \\
\hline \multicolumn{2}{|l|}{ Educational status ${ }^{\mathrm{b}}$} \\
\hline Unfinished secondary school & $20(47.6)$ \\
\hline Finished secondary school & $22(52.4)$ \\
\hline \multicolumn{2}{|l|}{ Occupational status ${ }^{\mathrm{b}}$} \\
\hline Working & $13(31.0)$ \\
\hline Unemployed & $10(23.8)$ \\
\hline Retired & $19(45.2)$ \\
\hline
\end{tabular}

Note: Values are in mean \pm SD or $\mathrm{n}(\%)$. SB, LPA, MVPA, and TPA are for an average day. CRF: cardiorespiratory fitness; DBP: diastolic blood pressure; FBG: fasting blood glucose; HDL-C: high-density lipoprotein cholesterol; IDF: International Diabetes Federation; LPA: light physical activity; MetS: metabolic syndrome; MVPA: moderate-to-vigorous physical activity; SB: sedentary behaviour; SBP: systolic blood pressure; TG: triglycerides; TPA: total physical activity, Waist: waist circumference.

a According to the International Diabetes Federation criteria.

b Missing data. Reasons: Incomplete patient medical record data for illness duration and chlorpromazine equivalent dose ( $n=1$ and 3 , respectively); incomplete questionnaire data for severity of psychiatric symptoms, educational and occupational status (all $n=1$ ).

c Severity of psychiatric symptoms was assessed using the Brief Symptoms Inventory18 ; with higher scores indicating high severity of psychiatric symptoms.
PA, moderate-to-vigorous PA, and CRF groups compared with their counterparts; however, significance was only indicated in diastolic blood pressure between SB levels and in waist and triglycerides between light PA levels (all $\mathrm{p}<0.05$ ). The low total PA group had a significantly higher waist than the high total PA group $(\mathrm{p}=0.025)$. Additionally, a heightened CCRS was found in the high SB, and low PA (all variables) and CRF groups, albeit significant only when comparing light and total PA levels (both $\mathrm{p}<0.05$; data not shown).

Correlations between SB, PA, CRF, and cardiometabolic risk are presented in Table 3. SB had a significant negative correlation with all PA variables and CRF (all $\mathrm{p}<0.05$ ). Furthermore, moderate-to-vigorous and total PA had a significant moderate positive correlation with CRF (both $\mathrm{p}<0.05$ ). The CCRS and the number of cardiometabolic abnormalities were positively associated with SB and negatively associated with light PA, total PA, and CRF (all p $<0.05$ ). In general, significant correlations with individual-cardiometabolic risk factors were fair to moderate, ranging from 0.30 to 0.57 , expressed in absolute terms (Table 3 ).

Due to missing data, a subsample of 38 patients ( 32 men and 11 with bipolar disorders) with no changes in the correlation coefficients of the significant correlates (all $\mathrm{p}<0.05$; data not shown) was included in the multiple regression analysis (Table 4). SB was associated with the CCRS ( $\beta=0.28, p=0.049)$, the number of cardiometabolic abnormalities $(\beta=0.33, p=0.023)$, waist $(\beta=0.45, p=0.001)$, and fasting blood glucose $(\beta=0.38, p=0.023)$ (Model- 1$)$. The associations with the CCRS and the number of cardiometabolic abnormalities became non-significant after adjustment for the combined PA and CRF. A significant association for waist remained after adjusting for moderate-tovigorous PA and CRF. Furthermore, the association with fasting blood glucose remained significant after adjusting for all PA variables, in separate fully adjusted models, and for CRF. Light PA was associated with waist $(\beta=0.40, p=0.005)$ (Model- 1 ). The association attenuated to non-significance after adjusting for SB, moderate-tovigorous PA, and CRF. Moderate-to-vigorous PA was associated with the CCRS $(\beta=-0.30, p=0.043$ ) (Model- 1 ). The association moved beyond the threshold of significance after adjusting for SB, PA (light and total PA, in separate fully adjusted models), and CRF. Total PA was associated with the CCRS $(\beta=-0.32, \mathrm{p}=0.024)$ and waist ( $\beta=-0.38, p=0.008)$ (Model-1). These results became nonsignificant after adjust for SB, moderate-to-vigorous PA, and CRF. CRF was significantly associated with the CCRS $(\beta=-0.42, \mathrm{p}=0.026)$ (Model 2), waist $(\beta=-0.55, \mathrm{p}=0.001)$, and systolic blood pressure $(\beta=-0.45, p=0.028)$ (Model-1). The associations with the CCRS and waist remained after adjusting for SB and all PA variables (in separate fully adjusted models) (Table 4 ).

\section{Discussion}

This is one of the few studies (Ekblom et al., 2015; Greer et al., 2015; Knaeps et al., 2016a; Knaeps et al., 2016b; Shuval et al., 2014; van der Velde et al., 2015) to evaluate the independent associations of SB, PA, and CRF with cardiometabolic risk, and the first to focus on patients with psychosis. The main result suggests that although high levels of SB and low levels of PA and CRF are associated with a higher clustered-cardiometabolic risk, only CRF remains significantly related independent of multiple confounders (including SB and PA). Additionally, when examining independently the single cardiometabolic risk factors, CRF and SB are associated with waist and SB with fasting blood glucose, and all of these associations are independent of the other potential exposures. Taken together, these results suggest the importance of considering CRF and SB, regardless of PA, in the prevention and treatment of metabolic disorders among patients with psychosis.

Our results showed the independent association between CRF and clustered-cardiometabolic risk consistent with aforementioned similar studies (Ekblom et al., 2015; Greer et al., 2015; Knaeps et al., 2016a; Knaeps et al., 2016b; Shuval et al., 2014; van der Velde et al., 2015), including three (Ekblom et al., 2015; Knaeps et al., 2016b; van der Velde 
Table 2

Comparison of cardiometabolic characteristics between levels of ST, PA, and CRF among outpatients with psychosis $(n=43)$.

\begin{tabular}{|c|c|c|c|c|c|c|c|c|c|c|}
\hline & \multicolumn{2}{|c|}{ SB (cut-off: 8.8 h/day) } & \multicolumn{2}{|c|}{ LPA (cut-off: 4.7 h/day) } & \multicolumn{2}{|c|}{ MVPA (cut-off: 1.7 h/day) } & \multicolumn{2}{|c|}{ TPA (cut-off: 6.3 h/day) } & \multicolumn{2}{|c|}{ CRF (cut-off: $607.2 \mathrm{~m}$ ) } \\
\hline & $\begin{array}{l}\text { High } \\
(n=21)\end{array}$ & $\begin{array}{l}\text { Low } \\
(n=22)\end{array}$ & $\begin{array}{l}\text { Low } \\
(n=21)\end{array}$ & $\begin{array}{l}\text { High } \\
(n=22)\end{array}$ & $\begin{array}{l}\text { Low } \\
(n=21)\end{array}$ & $\begin{array}{l}\text { High } \\
(n=22)\end{array}$ & $\begin{array}{l}\text { Low } \\
(n=21)\end{array}$ & $\begin{array}{l}\text { High } \\
(n=22)\end{array}$ & $\begin{array}{l}\text { Low } \\
(n=21)\end{array}$ & $\begin{array}{l}\text { High } \\
(n=22)\end{array}$ \\
\hline MetS $^{a}$ & $5(76.2)$ & $12(54.5)$ & $17(81.0)$ & $11(50.0)$ & $15(71.4)$ & $13(59.1)$ & $16(76.2)$ & $12(54.5)$ & $16(76.2)$ & $12(54.5)$ \\
\hline No. of meeting MetS ${ }^{a}$ & $3.3 \pm 1.7$ & $2.4 \pm 1.7$ & $3.4 \pm 1.4$ & $2.3 \pm 1.8$ & $3.2 \pm 1.7$ & $2.5 \pm 1.7$ & $3.1 \pm 1.7$ & $2.5 \pm 1.8$ & $3.2 \pm 1.7$ & $2.5 \pm 1.7$ \\
\hline Waist (cm) & $110.3 \pm 18.0$ & $101.2 \pm 14.0$ & $113.2 \pm 12.6$ & $98.4 \pm 16.8$ & $106.4 \pm 17.6$ & $104.8 \pm 15.8$ & $111.3 \pm 15.2$ & $100.1 \pm 16.2$ & $109.4 \pm 18.5$ & $102.0 \pm 13.8$ \\
\hline IDF-criteria & $19(90.5)$ & $17(77.3)$ & $21(100.0)$ & $15(68.2)$ & $19(90.5)$ & $17(77.3)$ & $20(95.2)$ & $16(72.7)$ & $19(90.5)$ & $17(77.3)$ \\
\hline $\mathrm{SBP}(\mathrm{mm} \mathrm{Hg})$ & $130.3 \pm 18.0$ & $122.5 \pm 17.2$ & $127.9 \pm 17.7$ & $124.8 \pm 18.2$ & $127.7 \pm 19.5$ & $125.0 \pm 16.4$ & $131.3 \pm 17.7$ & $121.5 \pm 16.9$ & $131.2 \pm 17.6$ & $121.6 \pm 17.1$ \\
\hline IDF-criteria & $12(57.1)$ & $9(40.9)$ & $11(52.4)$ & $10(45.5)$ & $12(57.1)$ & $9(40.9)$ & $13(61.9)$ & $8(36.4)$ & $13(61.9)$ & $8(36.4)$ \\
\hline $\mathrm{DBP}(\mathrm{mm} \mathrm{Hg})$ & $85.8 \pm 11.6$ & $79.1 \pm 9.6$ & $83.7 \pm 11.9$ & $81.2 \pm 10.3$ & $83.8 \pm 12.3$ & $81.1 \pm 9.7$ & $85.7 \pm 11.0$ & $79.2 \pm 10.3$ & $85.2 \pm 11.5$ & $79.7 \pm 10.1$ \\
\hline IDF-criteria & $13(61.9)$ & $7(31.8)$ & $9(42.9)$ & $11(50.0)$ & $12(57.1)$ & $8(36.4)$ & $11(52.4)$ & $9(40.9)$ & $11(52.4)$ & $9(40.9)$ \\
\hline $\mathrm{TG}(\mathrm{mg} / \mathrm{dL})$ & $231.3 \pm 196.6$ & $190.6 \pm 158.1$ & $215.4 \pm 102.9$ & $205.7 \pm 228.6$ & $233.5 \pm 198.5$ & $188.5 \pm 155.3$ & $208.7 \pm 101.3$ & $212.2 \pm 230.0$ & $209.7 \pm 144.7$ & $211.3 \pm 206.7$ \\
\hline IDF-criteria & $15(71.4)$ & $10(45.5)$ & $16(76.2)$ & $9(40.9)$ & $15(71.4)$ & $10(45.5)$ & $15(71.4)$ & $10(45.5)$ & $15(71.4)$ & $10(45.5)$ \\
\hline HDL-C (mg/dL) & $43.0 \pm 12.8$ & $45.7 \pm 11.0$ & $41.3 \pm 9.9$ & $47.3 \pm 13.0$ & $44.2 \pm 13.7$ & $44.6 \pm 10.1$ & $42.4 \pm 12.1$ & $46.3 \pm 11.6$ & $43.7 \pm 11.7$ & $45.1 \pm 12.2$ \\
\hline IDF-criteria & $10(47.6)$ & $11(50.0)$ & $11(52.4)$ & $10(45.5)$ & $11(52.4)$ & $10(45.5)$ & $11(52.4)$ & $10(45.5)$ & $11(52.4)$ & $10(45.5)$ \\
\hline $\mathrm{FBG}(\mathrm{mg} / \mathrm{dL})$ & $117.0 \pm 51.2$ & $97.2 \pm 17.8$ & $110.7 \pm 527.2$ & $103.2 \pm 38.1$ & $113.9 \pm 51.7$ & $100.2 \pm 19.4$ & $106.7 \pm 40.8$ & $107.0 \pm 37.9$ & $112.4 \pm 41.9$ & $101.5 \pm 35.9$ \\
\hline IDF-criteria & $12(57.1)$ & $8(36.4)$ & $11(52.4)$ & $9(40.9)$ & $11(52.4)$ & 9 (40.9) & $9(42.9)$ & $11(50.0)$ & $11(52.4)$ & $9(40.9)$ \\
\hline
\end{tabular}

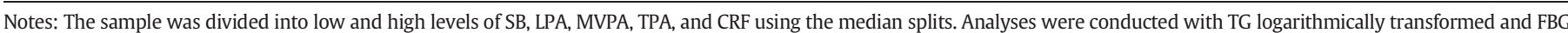

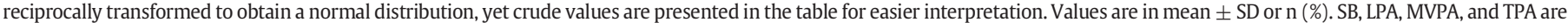
for an average day.

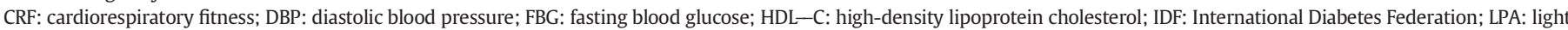

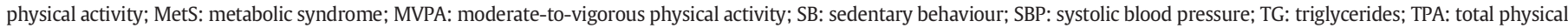
activity, Waist: waist circumference.

Boldface indicates statistical significance ( $\mathrm{p}$-value $<0.05$ ).

a According to the International Diabetes Federation-criteria.

et al., 2015) that objectively measured SB and PA and two (Knaeps et al., 2016a; Knaeps et al., 2016b) that used a clustering of individualcardiometabolic risk factors in the same individual that might reflect cardiometabolic risk even better than single independent risk factors, as well as the number of cardiometabolic abnormalities and metabolic syndrome (Wijndaele et al., 2006). Our results in patients with psychosis indicated that, together with the lack of association of SB and PA, CRF is the most important exposure for cardiometabolic risk, concurring with the findings of the two studies that used the CCRS (Knaeps et al., 2016a; Knaeps et al., 2016b). An explanation may be because CRF reflects both participation in sedentary and physical activities and the state of physiological systems, thereby providing more information about health status. Moreover, each $5-\mathrm{mL} \cdot \mathrm{kg}^{-1} \cdot \mathrm{min}^{-1}$ decrement in peak oxygen uptake (the criterion measure of CRF) corresponds to $56 \%$ higher prevalence of cardiovascular risk factors (Aspenes et al., 2011), being a deficient CRF one of the main cardiovascular mortality risk factors (Harber et al., 2017). These findings are of clinical interest, and, consistent with strongly supported evidence (Ross et al., 2016), highlight that CRF must be considered as a vital sign in clinical practice and in public health.

No study has accounted for SB, PA, and CRF simultaneously in patients with psychosis, and consequently, our results can be compared only with studies including two of the three exposures. Consistent findings in patients with psychosis reported that only CRF, and not PA, was significantly correlated with clustered-cardiometabolic risk (metabolic syndrome) (Nyboe et al., 2015). In another study (Stubbs et al., 2015), it was found that SB, and not PA, was associated with high-sensitivity C-reactive protein level, an inflammatory-marker associated with metabolic syndrome (Kazemi-Bajestani et al., 2017). Although similar findings, the two aforementioned studies (Nyboe et al., 2015; Stubbs et al., 2015) were based on self-reported measures of SB and PA, and consequently, direct comparisons with our results may not be valid. Therefore, in addition to examining the relationship of $\mathrm{SB}, \mathrm{PA}$ and CRF together for predicting clustered-cardiometabolic risk, our work contributes to the knowledge by objectively measuring behaviour.

Table 3

Pearson correlation coefficients $(r)$ for the association between SB, PA, CRF, and cardiometabolic risk among outpatients with psychosis ( $n=43$ ).

\begin{tabular}{|c|c|c|c|c|c|c|c|c|c|c|}
\hline & \multicolumn{2}{|l|}{ SB } & \multicolumn{2}{|l|}{ LPA } & \multicolumn{2}{|l|}{ MVPA } & \multicolumn{2}{|l|}{ TPA } & \multicolumn{2}{|l|}{ CRF } \\
\hline & $r$ & p-Value & $r$ & p-Value & $r$ & p-Value & $r$ & p-Value & $r$ & p-Value \\
\hline CCRS & 0.43 & 0.004 & -0.45 & 0.002 & -0.27 & 0.084 & -0.39 & 0.009 & -0.45 & 0.003 \\
\hline No. of meeting MetS ${ }^{a}$ & 0.38 & 0.012 & -0.41 & 0.006 & -0.23 & 0.130 & -0.40 & 0.004 & -0.35 & 0.021 \\
\hline Waist & 0.51 & 0.001 & -0.57 & $<0.001$ & -0.25 & 0.111 & -0.47 & $<0.001$ & -0.34 & 0.025 \\
\hline SBP & 0.34 & 0.026 & -0.25 & 0.101 & -0.17 & 0.280 & -0.24 & 0.060 & -0.38 & 0.012 \\
\hline DBP & 0.34 & 0.026 & -0.28 & 0.066 & -0.08 & 0.618 & -0.24 & 0.061 & -0.25 & 0.099 \\
\hline TG & -0.18 & 0.258 & 0.24 & 0.123 & 0.17 & 0.277 & 0.25 & 0.051 & 0.17 & 0.270 \\
\hline HDL-C & 0.20 & 0.203 & -0.20 & 0.188 & -0.21 & 0.182 & -0.30 & 0.025 & -0.26 & 0.088 \\
\hline FBG & 0.39 & 0.010 & -0.10 & 0.522 & 0.06 & 0.722 & -0.24 & 0.120 & -0.07 & 0.677 \\
\hline SB & 1.00 & - & -0.60 & $<0.001$ & -0.55 & $<\mathbf{0 . 0 0 1}$ & -0.67 & $<\mathbf{0 . 0 0 1}$ & -0.37 & 0.014 \\
\hline LPA & -0.60 & $<0.001$ & 1.00 & - & 0.45 & 0.003 & 0.88 & $<0.001$ & 0.24 & 0.126 \\
\hline MVPA & -0.55 & $<0.001$ & 0.45 & 0.003 & 1.00 & - & 0.78 & $<0.001$ & 0.53 & $<0.001$ \\
\hline TPA & -0.67 & $<0.001$ & 0.88 & $<0.001$ & 0.78 & $<0.001$ & 1.00 & - & 0.47 & 0.001 \\
\hline CRF & -0.37 & 0.014 & 0.24 & 0.126 & 0.53 & $<\mathbf{0 . 0 0 1}$ & 0.47 & $<0.001$ & 1.00 & - \\
\hline
\end{tabular}

Notes: Analyses were conducted with MVPA and TG logarithmically transformed, and FBG reciprocally transformed, to obtain a normal distribution.

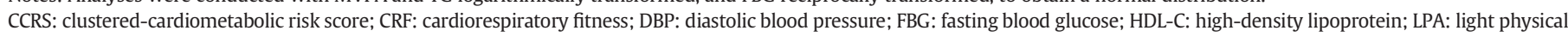

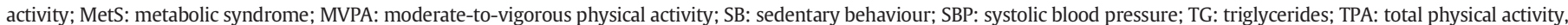
Waist: waist circumference.

Boldface indicates statistical significance ( $\mathrm{p}$-value $<0.05$ ).

a According to the International Diabetes Federation-criteria (cita). 
Table 4

Standardized regression coefficients $(\beta)$ of SB, PA, and CRF for cardiometabolic risk among patients with psychosis $(n=38)$.

\begin{tabular}{|c|c|c|c|c|c|c|c|c|c|c|c|c|c|c|c|c|c|}
\hline & & \multicolumn{2}{|l|}{ CCRS } & \multicolumn{2}{|c|}{$\begin{array}{l}\text { No. of meeting } \\
\text { MetS }^{\mathrm{a}}\end{array}$} & \multicolumn{2}{|c|}{ Waist $(\mathrm{cm})$} & \multicolumn{2}{|c|}{$\mathrm{SBP}(\mathrm{mm} \mathrm{Hg})$} & \multicolumn{2}{|c|}{$\mathrm{DBP}(\mathrm{mm} \mathrm{Hg})$} & \multicolumn{2}{|c|}{ TG (mg/dL) } & \multicolumn{2}{|c|}{ HDL-C (mg/dL) } & \multicolumn{2}{|c|}{$\mathrm{FBG}(\mathrm{mg} / \mathrm{dL})$} \\
\hline & & $\beta$ & p-Value & $\beta$ & p-Value & $\beta$ & p-Value & $\beta$ & p-Value & $\beta$ & p-Value & $\beta$ & p-Value & $\beta$ & p-Value & $\beta$ & p-Value \\
\hline \multirow[t]{9}{*}{ SB } & M1 & 0.28 & 0.049 & 0.33 & 0.023 & 0.45 & 0.001 & 0.26 & 0.128 & 0.29 & 0.090 & 0.20 & 0.198 & -0.16 & 0.297 & 0.38 & 0.023 \\
\hline & M2 & & & & & & & 0.01 & 0.967 & -0.01 & 0.945 & 0.24 & 0.202 & -0.09 & 0.642 & 0.44 & 0.032 \\
\hline & $\mathrm{M} 2+\mathrm{LPA}$ & 0.34 & 0.042 & 0.29 & 0.108 & 0.34 & 0.038 & 0.01 & 0.960 & -0.01 & 0.979 & 0.30 & 0.159 & -0.11 & 0.595 & 0.59 & 0.010 \\
\hline & $\mathrm{M} 2$ + MVPA & 0.22 & 0.104 & 0.35 & 0.072 & 0.48 & 0.007 & 0.01 & 0.953 & 0.06 & 0.805 & 0.16 & 0.495 & 0.05 & 0.824 & 0.77 & 0.002 \\
\hline & $\mathrm{M} 2+\mathrm{TPA}$ & 0.30 & 0.099 & 0.31 & 0.128 & 0.38 & 0.040 & 0.04 & 0.864 & 0.01 & 0.966 & 0.20 & 0.397 & -0.02 & 0.921 & 0.71 & 0.005 \\
\hline & $\mathrm{M} 2+\mathrm{CRF}$ & 0.19 & 0.124 & 0.28 & 0.086 & 0.31 & 0.026 & -0.04 & 0.848 & -0.02 & 0.895 & 0.21 & 0.278 & -0.04 & 0.845 & 0.43 & 0.045 \\
\hline & $\mathrm{M} 2+\mathrm{LPA}+\mathrm{CRF}$ & 0.22 & 0.179 & 0.26 & 0.189 & 0.22 & 0.161 & -0.03 & 0.888 & -0.02 & 0.936 & 0.28 & 0.212 & -0.07 & 0.757 & 0.58 & 0.015 \\
\hline & $\mathrm{M} 2+\mathrm{MVPA}+\mathrm{CRF}$ & 0.19 & 0.147 & 0.32 & 0.107 & 0.40 & 0.016 & 0.02 & 0.938 & 0.06 & 0.801 & 0.16 & 0.498 & 0.05 & 0.836 & 0.77 & 0.002 \\
\hline & $\mathrm{M} 2+\mathrm{TPA}+\mathrm{CRF}$ & 0.24 & 0.166 & 0.29 & 0.164 & 0.32 & 0.069 & 0.04 & 0.880 & 0.01 & 0.971 & 0.20 & 0.409 & -0.02 & 0.935 & 0.71 & 0.005 \\
\hline \multirow[t]{6}{*}{ LPA } & M1 & -0.26 & 0.063 & -0.24 & 0.109 & -0.40 & 0.005 & -0.22 & 0.197 & -0.25 & 0.150 & -0.03 & 0.836 & 0.09 & 0.585 & -0.03 & 0.851 \\
\hline & M2 & & & & & & & 0.00 & 0.994 & 0.02 & 0.922 & 0.00 & 0.983 & -0.01 & 0.977 & 0.05 & 0.797 \\
\hline & $\mathrm{M} 2+\mathrm{SB}$ & -0.05 & 0.758 & -0.06 & 0.745 & -0.19 & 0.230 & 0.01 & 0.976 & 0.01 & 0.942 & 0.13 & 0.517 & -0.06 & 0.784 & 0.32 & 0.134 \\
\hline & $\mathrm{M} 2+\mathrm{MVPA}$ & -0.15 & 0.381 & -0.18 & 0.316 & -0.36 & 0.035 & 0.00 & 0.998 & -0.03 & 0.886 & 0.14 & 0.498 & -0.13 & 0.513 & 0.02 & 0.933 \\
\hline & $\mathrm{M} 2+\mathrm{CRF}$ & -0.12 & 0.356 & -0.18 & 0.268 & -0.27 & 0.045 & 0.03 & 0.888 & 0.02 & 0.895 & 0.02 & 0.920 & -0.03 & 0.849 & 0.07 & 0.722 \\
\hline & $\mathrm{M} 2+\mathrm{SB}+\mathrm{MVPA}+\mathrm{CRF}$ & -0.03 & 0.867 & -0.07 & 0.718 & 0.25 & 0.163 & -0.02 & 0.940 & -0.03 & 0.897 & 0.18 & 0.420 & -0.12 & 0.590 & 0.17 & 0.423 \\
\hline \multirow[t]{8}{*}{ MVPA } & M1 & -0.30 & 0.043 & -0.21 & 0.178 & -0.29 & 0.061 & -0.16 & 0.379 & -0.13 & 0.497 & -0.21 & 0.200 & 0.23 & 0.161 & 0.01 & 0.954 \\
\hline & M2 & & & & & & & 0.00 & 0.991 & 0.07 & 0.657 & -0.21 & 0.229 & 0.18 & 0.287 & 0.07 & 0.713 \\
\hline & $\mathrm{M} 2+\mathrm{LPA}$ & -0.21 & 0.242 & -0.10 & 0.599 & -0.07 & 0.689 & 0.00 & 0.994 & 0.09 & 0.654 & -0.28 & 0.174 & 0.20 & 0.442 & 0.06 & 0.792 \\
\hline & $\mathrm{M} 2+\mathrm{SB}$ & -0.25 & 0.093 & 0.03 & 0.873 & 0.05 & 0.776 & 0.01 & 0.965 & 0.11 & 0.620 & -0.12 & 0.588 & 0.21 & 0.334 & 0.51 & 0.023 \\
\hline & $\mathrm{M} 2+\mathrm{TPA}$ & -0.08 & 0.762 & 0.01 & 0.980 & 0.12 & 0.644 & -0.06 & 0.845 & 0.14 & 0.615 & -0.21 & 0.491 & 0.25 & 0.217 & 0.15 & 0.669 \\
\hline & $\mathrm{M} 2+\mathrm{CRF}$ & -0.10 & 0.536 & -0.12 & 0.529 & -0.06 & 0.717 & 0.09 & 0.654 & 0.11 & 0.549 & -0.18 & 0.362 & 0.12 & 0.518 & 0.16 & 0.468 \\
\hline & $\mathrm{M} 2+\mathrm{SB}+\mathrm{LPA}+\mathrm{CRF}$ & 0.07 & 0.722 & 0.10 & 0.660 & 0.25 & 0.163 & 0.10 & 0.690 & 0.16 & 0.534 & -0.15 & 0.550 & 0.31 & 0.239 & 0.54 & 0.033 \\
\hline & $\mathrm{M} 2+\mathrm{SB}+\mathrm{TPA}+\mathrm{CRF}$ & 0.07 & 0.761 & 0.12 & 0.677 & 0.30 & 0.197 & 0.02 & 0.943 & 0.19 & 0.550 & -0.12 & 0.704 & 0.26 & 0.403 & 0.48 & 0.122 \\
\hline \multirow[t]{6}{*}{ TPA } & M1 & -0.32 & 0.024 & -0.25 & 0.097 & -0.38 & 0.008 & -0.19 & 0.277 & -0.23 & 0.197 & -0.17 & 0.290 & 0.17 & 0.268 & -0.05 & 0.784 \\
\hline & M2 & & & & & & & 0.03 & 0.872 & 0.03 & 0.863 & -0.18 & 0.329 & 0.11 & 0.524 & 0.03 & 0.898 \\
\hline & $\mathrm{M} 2+\mathrm{SB}$ & -0.10 & 0.586 & -0.03 & 0.888 & -0.11 & 0.545 & 0.05 & 0.819 & 0.04 & 0.872 & -0.06 & 0.791 & 0.10 & 0.660 & 0.44 & 0.057 \\
\hline & $\mathrm{M} 2+\mathrm{MVPA}$ & -0.26 & 0.295 & -0.26 & 0.342 & -0.48 & 0.061 & 0.08 & 0.802 & -0.09 & 0.761 & -0.01 & 0.980 & -0.11 & 0.720 & -0.09 & 0.788 \\
\hline & $\mathrm{M} 2+\mathrm{CRF}$ & -0.13 & 0.389 & -0.17 & 0.332 & -0.19 & 0.214 & 0.11 & 0.576 & 0.05 & 0.775 & -0.13 & 0.609 & 0.04 & 0.823 & 0.09 & 0.671 \\
\hline & $\mathrm{M} 2+\mathrm{SB}+\mathrm{MVPA}+\mathrm{CRF}$ & -0.09 & 0.700 & -0.06 & 0.821 & -0.18 & 0.436 & 0.11 & 0.724 & -0.06 & 0.839 & 0.06 & 0.860 & -0.12 & 0.715 & 0.19 & 0.534 \\
\hline \multirow[t]{9}{*}{ CRF } & M1 & -0.42 & 0.026 & -0.29 & 0.118 & -0.55 & 0.001 & -0.45 & 0.028 & -0.40 & 0.058 & -0.18 & 0.350 & 0.30 & 0.113 & -0.22 & 0.296 \\
\hline & M2 & & & & & & & -0.21 & 0.359 & -0.05 & 0.807 & -0.20 & 0.393 & 0.25 & 0.265 & -0.18 & 0.492 \\
\hline & $\mathrm{M} 2+\mathrm{SB}$ & -0.46 & 0.005 & -0.12 & 0.553 & -0.37 & 0.034 & -0.22 & 0.357 & -0.06 & 0.791 & -0.13 & 0.575 & 0.24 & 0.311 & -0.05 & 0.850 \\
\hline & $\mathrm{M} 2+\mathrm{LPA}$ & -0.45 & 0.010 & -0.21 & 0.293 & -0.43 & 0.011 & -0.21 & 0.362 & -0.06 & 0.798 & -0.20 & 0.399 & 0.26 & 0.267 & -0.19 & 0.472 \\
\hline & $\mathrm{M} 2+\mathrm{MVPA}$ & -0.45 & 0.018 & -0.22 & 0.317 & -0.52 & 0.009 & -0.26 & 0.314 & -0.12 & 0.634 & -0.10 & 0.708 & 0.18 & 0.470 & -0.27 & 0.355 \\
\hline & $\mathrm{M} 2+\mathrm{TPA}$ & -0.42 & 0.026 & -0.17 & 0.423 & -0.43 & 0.026 & -0.26 & 0.294 & -0.08 & 0.740 & -0.13 & 0.609 & 0.23 & 0.355 & -0.23 & 0.431 \\
\hline & $\mathrm{M} 2+\mathrm{SB}+\mathrm{LPA}$ & -0.37 & 0.039 & -0.11 & 0.583 & -0.35 & 0.045 & -0.22 & 0.366 & -0.06 & 0.793 & -0.14 & 0.565 & 0.24 & 0.315 & -0.06 & 0.808 \\
\hline & $\mathrm{M} 2+\mathrm{SB}+\mathrm{MVPA}$ & -0.42 & 0.026 & -0.14 & 0.505 & -0.42 & 0.021 & -0.26 & 0.323 & -0.12 & 0.637 & -0.10 & 0.701 & 0.18 & 0.481 & -0.29 & 0.250 \\
\hline & $\mathrm{M} 2+\mathrm{SB}+\mathrm{TPA}$ & -0.38 & 0.043 & -0.12 & 0.570 & -0.37 & 0.046 & -0.26 & 0.305 & -0.08 & 0.745 & -0.13 & 0.622 & 0.23 & 0.365 & -0.21 & 0.400 \\
\hline
\end{tabular}

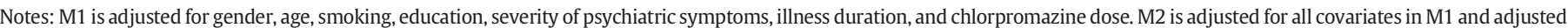

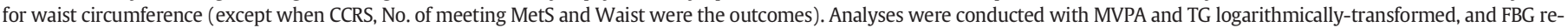
ciprocally-transformed, to obtain a normal distribution.

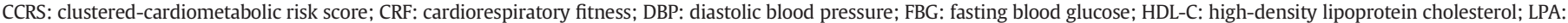

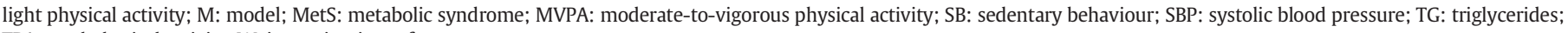
TPA: total physical activity, Waist: waist circumference.

Boldface indicates statistical significance ( $p$-value $<0.05$ ).

a According to the International Diabetes Federation-criteria.

Our results indicated that CRF and $\mathrm{SB}$ are both related to waist, independent from each other and from PA, suggesting that CRF and SB are two independent predictors of waist. Consistent findings in patients with psychosis have revealed significant associations between CRF and waist accounting for self-reported PA (Nyboe et al., 2015; Vancampfort et al., 2015a). However, our results were inconsistent with the findings of the only study in patients with psychosis that explored the SB-waist association adjusting for PA (Stubbs et al., 2017). This discrepancy may be because the authors of that study (Stubbs et al., 2017) used accelerometers to obtain an objective indirect estimations of SB and PA through the absence of whole-body movement and the number of steps, respectively, which could introduce bias for both exposures. Considering SB as the absence of whole-body movement is an important conceptual error (Sedentary Behaviour Research, 2012), and the number of steps only provides a value of total ambulatory PA, thereby excluding a range of free-living physical activities aside from walking and or running, such as gardening or washing, which have shown beneficial effects on cardiovascular health (van den Berg et al., 2010). Accordingly, the use of sensor combining physiological measures with movement and position sensing to identify SB and PA may be a more appropriate way to obtain accurate results.
Again, inconsistent with the aforementioned study (Stubbs et al., 2017), our results showed that the relationship between SB and fasting blood glucose remained significant when adjusting for PA. Discrepancies between studies can be explained, in addition to the different instruments of assessment of SB and PA used, because we assessed PA at different intensities and controlled for waist, a predisposing factor for the development of type 2 diabetes mellitus (Freemantle et al., 2008), showing that the SB-fasting blood glucose association did not vary. Additionally, our findings remained significant even when CRF was included with the rest of the confounders, suggesting that SB seems to be an important and independent risk factor for fasting blood glucose in patients with psychosis. However, further research combining SB, PA, and CRF interactions with cardiometabolic outcomes in patients with psychosis is needed before any firm conclusions can be made.

The major strength of the study is the objective measurement of SB and PA using strict inclusion criteria. All patients wore the SenseWear for seven consecutive days with at least $1368 \mathrm{~min} /$ day, and the Hawthorne effect (explained in brief in the method section) was minimized. Another strength is the use of the time spent in different intensities of PA which extends the knowledge on the independent association of $\mathrm{SB}, \mathrm{PA}$, and CRF with cardiometabolic risk and informs, in greater detail, 
on intervention strategies for treating metabolic disorders among patients with psychosis focused on the development of a more active lifestyle that enhance/maintain CRF. Finally, the adjustment for covariates that could influence the relationships between SB, PA, and CRF with cardiometabolic risk can also be considered a strength. Multiple linear regression analyses were adjusted for obesity in addition to other covariates such as gender, age, smoking, education, symptom severity, illness duration, and antipsychotic medication, previously controlled in psychiatric patients studies (i.e., Stubbs et al., 2017). This approach was only applied in two (Shuval et al., 2014; van der Velde et al., 2015) of the six studies (Ekblom et al., 2015; Greer et al., 2015; Knaeps et al., 2016a; Knaeps et al., 2016b; Shuval et al., 2014; van der Velde et al., 2015) that evaluated the independent associations of SB, $\mathrm{PA}$, and CRF with cardiometabolic risk.

Some limitations should be noted. The small sample size of outpatients, predominantly men diagnosed with schizophrenia, may limit the generalization to other groups. Future research should use large and homogeneous sample, and compare between different clinical settings, genders, and psychiatric disorders. Another limitation is that the current study was cross-sectional in design. Longitudinal studies are needed to identify any casual relationships. Although the absence of control group can be considered a study limitation, we compared our data against all published studies that have examined the independent associations of SB, PA, and CRF with cardiometabolic risk, each of which is based on observations from hundreds of healthy individuals. Nevertheless, further studies including control group are required to confirm or refute our findings. The SenseWear cannot differentiate body positions, and consequently, standing may be considered as SB. However, it may solve limitations presented by accelerometers and inclinometers through heat production measurements and placement on the upper arm. Additionally, the SenseWear underestimates energy expenditure at higher PA intensities (Drenowatz and Eisenmann, 2011). However, because we used time engaged in moderate-to-vigorous PA, it is unlikely that this affected our results. Moreover, because the objective measurement did not inform about the type of SB and PA, future studies should combine objective with self-report measurements. In patients with severe mental illness, the Sedentary Behaviour and the International Physical Activity questionnaires seem appropriate for quantifying time engaged in different SBs (Bueno-Antequera et al., 2017) and PAs (Faulkner et al., 2006), respectively. The assessment of CRF using an indirect measurement and a submaximal test could be considered as another limitation. However, the test used in this study has been found to be a reliable and valid method in patients with psychosis (Gomes et al., 2016). The CCRS has several advantages for evaluating cardiometabolic risk (Wijndaele et al., 2006), is sample-specific and is based on the assumption that each component is weighted equally in predicting cardiometabolic risk. Finally, dietary information was not considered as a covariate, and the data on symptomatology was self-reported.

In conclusion, low CRF was found to be a predictor of high clusteredcardiometabolic risk independent of multiple confounders, including SB and $\mathrm{PA}$, in patients with psychosis. This study further found associations of SB, PA, and CRF with individual-cardiometabolic risk factors. Therefore, in addition to developing interventions to reduce SB and increase $\mathrm{PA}$, interventions of randomized controlled trials of physical exercise in patients with psychosis are needed to determine whether reduced CRF and increased cardiometabolic risk can be improved.

\section{Acknowledgement}

The authors gratefully acknowledge all patients for their collaboration. We also acknowledge the health clinic members involved in the recruitment for their effort and great enthusiasm.

\section{Contributors}

JB and DM designed the study and wrote the protocol. All authors were responsible for the acquisition of the data. JB and DM performed the statistical analyses and JB wrote the manuscript. All authors provided critical review of the manuscript and approved the final version.

\section{Role of the funding source}

The work was funded by Research Group CTS-948, Universidad Pablo de Olavide Andalusian Government, European University of Madrid, Cátedra Real Madrid, Spain (funding project number P2017/RM08), Biomedical Research Networking Center on Frailty and Healthy Aging (CIBERFES) and FEDER funds from the European Union (CB16/10/00477). J.B. is supported by the Spanish Ministry of Education (grant number FPU13/05130). The funders had no role in study design; collection, analysis, and interpretation of data; writing the report; and the decision to submit the report for publication.

\section{References}

Alberti, K.G., Zimmet, P., Shaw, J., 2006. Metabolic syndrome-a new world-wide definition. A consensus statement from the International Diabetes Federation. Diabet. Med. 23 (5), 469-480.

Aspenes, S.T., Nilsen, T.I., Skaug, E.A., Bertheussen, G.F., Ellingsen, O., Vatten, L., Wisloff, U, 2011. Peak oxygen uptake and cardiovascular risk factors in 4631 healthy women and men. Med. Sci. Sports Exerc. 43 (8), 1465-1473.

van den Berg, A.E., van Winsum-Westra, M., de Vries, S., van Dillen, S.M., 2010. Allotment gardening and health: a comparative survey among allotment gardeners and their neighbors without an allotment. Environ. Health 9, 74.

Biswas, A., Oh, P.I., Faulkner, G.E., Bajaj, R.R., Silver, M.A., Mitchell, M.S., Alter, D.A., 2015 Sedentary time and its association with risk for disease incidence, mortality, and hospitalization in adults: a systematic review and meta-analysis. Ann. Intern. Med. 162 (2), 123-132.

Bruins, J., Pijnenborg, G.H., van den Heuvel, E.R., Visser, E., Corpeleijn, E., Bartels-Velthuis, A.A., Bruggeman, R., Jorg, F., 2017. Persistent low rates of treatment of metabolic risk factors in people with psychotic disorders: a PHAMOUS study. J. Clin. Psychiatry.

Bueno-Antequera, J., Oviedo-Caro, M.A., Munguia-Izquierdo, D., 2017. Sedentary behaviour patterns in outpatients with severe mental illness: a cross-sectional study using objective and self-reported methods. The PsychiActive project. Psychiatry Res. $255,146-152$

Corder, K., Ekelund, U., Steele, R.M., Wareham, N.J., Brage, S., 2008. Assessment of physical activity in youth. J. Appl. Physiol. (1985) 105 (3), 977-987.

Correll, C.U., Solmi, M., Veronese, N., Bortolato, B., Rosson, S., Santonastaso, P., ThapaChhetri, N., Fornaro, M., Gallicchio, D., Collantoni, E., Pigato, G., Favaro, A., Monaco, F., Kohler, C., Vancampfort, D., Ward, P.B., Gaughran, F., Carvalho, A.F., Stubbs, B., 2017. Prevalence, incidence and mortality from cardiovascular disease in patients with pooled and specific severe mental illness: a large-scale meta-analysis of 3,211,768 patients and 113,383,368 controls. World Psychiatry 16 (2), 163-180.

Derogatis, L., 2001. Brief Symptom Inventory (BSI)-18: Administration, Scoring and Procedures Manual. NCS Pearson, Inc., Minneapolis.

Drenowatz, C., Eisenmann, J.C., 2011. Validation of the SenseWear Armband at high intensity exercise. Eur. J. Appl. Physiol. 111 (5), 883-887.

Ekblom, O., Ekblom-Bak, E., Rosengren, A., Hallsten, M., Bergstrom, G., Borjesson, M., 2015 Cardiorespiratory fitness, sedentary behaviour and physical activity are independently associated with the metabolic syndrome, results from the SCAPIS pilot study. PLoS One 10 (6), e0131586.

Faulkner, G., Cohn, T., Remington, G., 2006. Validation of a physical activity assessment tool for individuals with schizophrenia. Schizophr. Res. 82 (2-3), 225-231.

Freemantle, N., Holmes, J., Hockey, A., Kumar, S., 2008. How strong is the association between abdominal obesity and the incidence of type 2 diabetes? Int. J. Clin. Pract. 62 (9), 1391-1396.

Gardner, D.M., Murphy, A.L., O'Donnell, H., Centorrino, F., Baldessarini, R.J., 2010. International consensus study of antipsychotic dosing. Am. J. Psychiatry 167 (6), 686-693.

Gomes, E., Bastos, T., Probst, M., Ribeiro, J.C., Silva, G., Corredeira, R., 2016. Reliability and validity of 6MWT for outpatients with schizophrenia: a preliminary study. Psychiatry Res. 237, 37-42

Greer, A.E., Sui, X., Maslow, A.L., Greer, B.K., Blair, S.N., 2015. The effects of sedentary behavior on metabolic syndrome independent of physical activity and cardiorespiratory fitness. J. Phys. Act. Health 12 (1), 68-73.

Harber, M.P., Kaminsky, L.A., Arena, R., Blair, S.N., Franklin, B.A., Myers, J., Ross, R., 2017 Impact of cardiorespiratory fitness on all-cause and disease-specific mortality: advances since 2009. Prog. Cardiovasc. Dis. 60 (1), 11-20.

Johannsen, D.L., Calabro, M.A., Stewart, J., Franke, W., Rood, J.C., Welk, G.J., 2010. Accuracy of armband monitors for measuring daily energy expenditure in healthy adults. Med. Sci. Sports Exerc. 42 (11), 2134-2140.

Kazemi-Bajestani, S.M., Tayefi, M., Ebrahimi, M., Heidari-Bakavoli, A.R., Moohebati, M. Parizadeh, S.M., Esmaeili, H., Ferns, G.A., Ghayour-Mobarhan, M., 2017. The prevalence of metabolic syndrome increases with serum high sensitivity C-reactive protein concentration in individuals without a history of cardiovascular disease: a report from a large Persian cohort. Ann. Clin. Biochem. 4563216676842.

Knaeps, S., Bourgois, J.G., Charlier, R., Mertens, E., Lefevre, J., Wijndaele, K., 2016a. Ten-year change in sedentary behaviour, moderate-to-vigorous physical activity, cardiorespiratory fitness and cardiometabolic risk: independent associations and mediation analysis. Br. J. Sports Med.

Knaeps, S., Lefevre, J., Wijtzes, A., Charlier, R., Mertens, E., Bourgois, J.G., 2016b. Independent associations between sedentary time, moderate-to-vigorous physical activity, cardiorespiratory fitness and cardio-metabolic health: a cross-sectional study. PLoS One 11 (7), e0160166.

Lawrence, D., Hancock, K.J., Kisely, S., 2013. The gap in life expectancy from preventable physical illness in psychiatric patients in Western Australia: retrospective analysis of population based registers. BMJ 346, f2539.

Montgomery, D.C., Peck, E.A., Vining, G.G., 2012. Introduction to Linear Regression Analy sis. 5th ed. Wiley, Hoboken, NJ. 
Nyboe, L., Vestergaard, C.H., Moeller, M.K., Lund, H., Videbech, P., 2015. Metabolic syndrome and aerobic fitness in patients with first-episode schizophrenia, including a 1-year follow-up. Schizophr. Res. 168 (1-2), 381-387.

Prinz, U., Nutzinger, D.O., Schulz, H., Petermann, F., Braukhaus, C., Andreas, S., 2013. Comparative psychometric analyses of the SCL-90-R and its short versions in patients with affective disorders. BMC Psychiatry 13, 104.

Rikli, R., Jones, C., 1999. Development and validation of a functional fitness test for community-residing older adults. J. Aging Phys. Act. 7, 127-161.

Ross, R., Blair, S.N., Arena, R., Church, T.S., Despres, J.P., Franklin, B.A., Haskell, W.L., Kaminsky, L.A., Levine, B.D., Lavie, C.J., Myers, J., Niebauer, J., Sallis, R., Sawada, S.S., Sui, X., Wisloff, U., 2016. Importance of assessing cardiorespiratory fitness in clinical practice: a case for fitness as a clinical vital sign: a scientific statement from the American Heart Association. Circulation 134 (24), 653-699.

Sedentary Behaviour Research, N, 2012. Letter to the editor: standardized use of the terms "sedentary" and "sedentary behaviours". Appl. Physiol. Nutr. Metab. 37 (3), 540-542.

Shuval, K., Finley, C.E., Barlow, C.E., Gabriel, K.P., Leonard, D., Kohl 3rd, H.W., 2014. Sedentary behavior, cardiorespiratory fitness, physical activity, and cardiometabolic risk in men: the cooper center longitudinal study. Mayo Clin. Proc. 89 (8), 1052-1062.

Soundy, A., Roskell, C., Stubbs, B., Vancampfort, D., 2014. Selection, use and psychometric properties of physical activity measures to assess individuals with severe mental illness: a narrative synthesis. Arch. Psychiatr. Nurs. 28 (2), 135-151.

Stubbs, B., Gardner-Sood, P., Smith, S., Ismail, K, Greenwood, K, Farmer, R, Gaughran, F, 2015. Sedentary behaviour is associated with elevated C-reactive protein levels in people with psychosis. Schizophr. Res. 168 (1-2), 461-464.

Stubbs, B., Firth, J., Berry, A., Schuch, F.B., Rosenbaum, S., Gaughran, F., Veronesse, N. Williams, J., Craig, T., Yung, A.R., Vancampfort, D., 2016a. How much physical activity do people with schizophrenia engage in? A systematic review, comparative metaanalysis and meta-regression. Schizophr. Res. 176 (2-3), 431-440.
Stubbs, B., Williams, J., Gaughran, F., Craig, T., 2016b. How sedentary are people with psychosis? A systematic review and meta-analysis. Schizophr. Res. 171 (1-3), 103-109.

Stubbs, B., Chen, L.J., Chung, M.S., Ku, P.W., 2017. Physical activity ameliorates the association between sedentary behavior and cardiometabolic risk among inpatients with schizophrenia: a comparison versus controls using accelerometry. Compr. Psychiatry $74,144-150$

Vancampfort, D., Guelinkcx, H., Probst, M., Stubbs, B., Rosenbaum, S., Ward, P.B., De Hert, M., 2015a. Associations between metabolic and aerobic fitness parameters in patients with schizophrenia. J. Nerv. Ment. Dis. 203 (1), 23-27.

Vancampfort, D., Stubbs, B., Mitchell, A.J., De Hert, M., Wampers, M., Ward, P.B., Rosenbaum, S., Correll, C.U., 2015b. Risk of metabolic syndrome and its components in people with schizophrenia and related psychotic disorders, bipolar disorder and major depressive disorder: a systematic review and meta-analysis. World Psychiatry 14 (3), 339-347.

Vancampfort, D., Rosenbaum, S., Schuch, F. Ward, P.B., Richards, J. Mugisha, J., Probst, M. Stubbs, B., 2017. Cardiorespiratory fitness in severe mental illness: a systematic review and meta-analysis. Sports Med. 47 (2), 343-352.

van der Velde, J.H., Savelberg, H.H., Schaper, N.C., Koster, A., 2015. Moderate activity and fitness, not sedentary time, are independently associated with cardio-metabolic risk in U.S. adults aged 18-49. Int. J. Environ. Res. Public Health 12 (3), 2330-2343.

Ward, R., Anderson, G.S., 1998. Resilience of anthropometric data assembly strategies to imposed error. J. Sports Sci. 16 (8), 755-759.

Wijndaele, K., Beunen, G., Duvigneaud, N., Matton, L., Duquet, W., Thomis, M., Lefevre, J., Philippaerts, R.M., 2006. A continuous metabolic syndrome risk score: utility for epidemiological analyses. Diabetes Care 29 (10), 2329.

World Health Organization, 1992. The ICD-10 Classification of Mental and Behavioural Disorders. Clinical Descriptions and Diagnostic Guidelines. World Health Organization. 